\title{
THE MINIMAL SIZE OF A SQUARE WHICH INCLUDES A DIGITAL CONVEX $2 K-$ GON
}

\author{
Dragan M. Acketa and Joviša D. Žunić
}

This paper presents a construction of a digital convex $2 k$-gon (for a given natural number $k$ ), which can be inscribed into a square grid of the minimal possible size $F(2 k)$. An explicit expression for the function $F(2 k)$ is also given.

\section{INTRODUCTION}

Recently there have been a lot of papers which deal with some optimisation problems on digital shapes. One of such problems will be studied here. It is related to convexity, one of the basic computational geometry properties [8].

A digital convex polygon is a polygon, all the vertices of which are points on the integer grid and the interior angles of which are strictly less than $\pi$ radians. The diameter of a digital convex polygon is the minimal edge size of the circumscribed digital square with the edges parallel to the coordinate axes.

This paper deals with the following optimisation problem: Given a natural number $n$, determine the least possible natural number $m=F(n)$, or given $m$, determine the greatest possible natural number $n=G(m)$, such that there exists a digital convex polygon of diameter $m$ with $n$ edges. This problem is completely solved for $n$ even $(n=2 k$ ); an explicit expression for the function $F(2 k)$ is given and the construction of an optimal digital convex $2 k$-gon is described.

A similar problem: "what is the minimal possible area of a digital convex polygon with a given even number of vertices ?" - has been studied in [9]. A method for the construction of such a digital convex polygon has been given in the same paper.

Some of the other optimisation problems related to convexity on the integer grid have been considered in a number of recent papers (see, for example, $[1,2,3,4]$ ). Motivation for considering such questions comes from several sources, in particular from integer programming and computer graphics.

The relationship between the number of edges and the diameter of optimal digital convex polygons was studied in the papers $[10,2,3]$. In particular, two sequences $m(t)$ and $n(t)$ of natural numbers were introduced in [10], with the property that

Received 21st December, 1992

Copyright Clearance Centre, Inc. Serial-fee code: 0004-9729/93 $\$ \mathbf{A 2 . 0 0 + 0 . 0 0}$. 
$F(n(t))=m(t)$; this means that there exists the corresponding optimal digital convex $n(t)$-gon $P(t)$ with diameter $m(t)$. An empirical approximation formula for $F(n)$ was derived in [10] as well. The exact formula for $F(n)$ was given in [2]. An almost optimal construction for general $n$ was described in [4]; that construction is almost optimal in the sense that the diameter of the constructed digital convex $n$-gon is not greater than $1+F(n)$.

If the number $k$ is even, then the construction of optimal digital $2 k$-gons is a generalisation of the construction of the polygons $P(t)$. Two families of auxiliary 6gons are introduced in order to cover the case when the number $k$ is odd.

\section{Preliminaries}

The diameter of a digital convex polygon $Q$ is equal to

$\max \left\{\max \left\{\left|x_{i}-x_{j}\right|,\left|y_{i}-y_{j}\right|\right\}\right.$, where $\left(\left(x_{i}, y_{i}\right),\left(x_{j}, y_{j}\right)\right)$ is a pair of vertices of $\left.Q\right\}$.

Note that the diameter is taken in the sense of the maximum distance.

Let $y_{\min }$ and $x_{\max }$ respectively denote the minimal $y$-coordinate and the maximal $x$-coordinate of the considered digital convex polygon $Q$. Generally, the $S E$-arc (southeast arc) of $Q$ is the sequence of consecutive edges $\left(V_{i}, V_{i+1}\right), 1 \leqslant i \leqslant k-1$, where:

- $V_{i}$ denotes a vertex $\left(x_{i}, y_{i}\right)$ of $Q$

- $x_{1}<\ldots<x_{k}=x_{\max } ; y_{\min }=y_{1}<\ldots<y_{k}$;

In particular, if the polygon $Q$ has a lower horizontal edge $\left(V_{0}, V_{1}\right)$ $\left(V_{0}=\left(x_{0}, y_{1}\right), V_{1}=\left(x_{1}, y_{1}\right), x_{0}<x_{1}\right)$, then this edge is additionally considered to be the first edge of the SE-arc. The NE-arc, the NW-arc and the SW-arc of a digital convex polygon are defined in the analogous way. (If the polygon $Q$ has a right vertical edge, then it is considered to be the first edge of the NE-arc, and so on.)

Given an edge $e=\left[\left(x_{1}, y_{1}\right),\left(x_{2}, y_{2}\right)\right]$ of a digital convex polygon, the edge slope of $e$ is defined to be the fraction:

$$
\frac{\left|x_{1}-x_{2}\right|}{\left|y_{1}-y_{2}\right|} \text { if } e \in \mathrm{NE} \text { - or SW-arc; } \quad \frac{\left|y_{1}-y_{2}\right|}{\left|x_{1}-x_{2}\right|} \text { if } e \in \mathrm{SE} \text { - or NW-arc, }
$$

while the bd-length of the edge $e$ is defined to be the sum $\left|x_{1}-x_{2}\right|+\left|y_{1}-y_{2}\right|$ (length in the sense of the block distance metrics).

A digital square $D S(p, q)$, where $p$ and $q$ are relatively prime natural numbers, is a digital convex 4-gon with the property that each arc has exactly one edge with the edge-slope $q / p$.

If the corresponding arcs of the two digital convex polygons $Q_{1}$ and $Q_{2}$ have no common edge slopes, then there exists a uniquely determined third digital convex 
polygon $Q_{3}$, called the sum (Minkowski sum) of $Q_{1}$ and $Q_{2}$. (For more details see [7, or 6]). Each arc of the polygon $Q_{3}$ includes all edges of the corresponding arcs of $Q_{1}$ and $Q_{2}$, sorted so that the convexity condition is preserved. If $Q_{3}$ is the sum of $Q_{1}$ and $Q_{2}$, then $Q_{2}$ is the difference of $Q_{3}$ and $Q_{1}$. The diameter of $Q_{3}$ is equal to the sum of the diameters of $Q_{1}$ and $Q_{2}$.

\section{GREEDY LOWER BOUND FOR $F(n)$}

A lower bound for $F(n)$, called the greedy lower bound and denoted by $g d l b(n)$, can be derived from the following observations:

The number $F(n)$ is equal to the minimal possible diameter of a digital convex $n$-gon. The diameter of a digital convex $n$-gon $P$ cannot be smaller than one fourth of the perimeter of $M R(P)$, where $M R(P)$ denotes the minimal rectangle with edges parallel to the axes, in which the polygon $P$ can be included.

This perimeter is equal to the sum of bd-lengths of edges of $P$ (Figure 1).

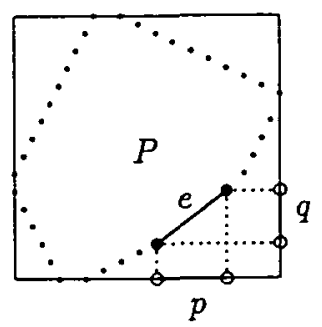

Figure 1. Orthogonal projections of the edges of $P$ exactly cover the perimeter of the rectangle $M R(P)$.

Consequently, a lower bound for $F(n)$ is equal to one quarter of the minimal possible sum Minsum( $n$ ) of bd-lengths of edges of a digital convex $n$-gon. We proceed with a calculation of Minsum(n):

Since the number of summands in Minsum(n) is fixed (equal to $n$ ), the minimisation requires the summands to be as small as possible. Such a choice of summands is naturally performed by the following "greedy" algorithm: choose as many summands equal to 1 as possible, then proceed with summands equal to 2 and so on. All these summands are of the form $(p+q)$, where $p / q$ is (a candidate for) an edge slope of an edge of $P$.

The following two rules must be obeyed by the edge slopes $p / q$ :

(a) the natural numbers $p$ and $q$ are relatively prime;

(b) each $p / q$ (with $p+q \geqslant 2$ ) can be used at most four times in $P$; that is, it has at most four associated summands $(p+q)$ in $\operatorname{Minsum}(n)$. 
Let $S(t)$ denote the set of all the different fractions $p / q$, where the natural numbers $p$ and $q$ are relatively prime and $2 \leqslant p+q \leqslant t$. A special family of optimal digital convex polygons $P(t)$ with $n(t)$ edges and diameter $m(t)$ can be constructed by applying the greedy algorithm. The polygon $P(t)$ is defined in the following way:

The edge slopes of edges of each arc of $P(t)$ are all the different fractions of the form $q / p$, where the natural numbers $p$ and $q$ are relatively prime and $p+q \leqslant t$. In addition, the edge slope of the first edge in each arc of $P(t)$ is equal to $0 / 1$. Thus the polygon $P(t)$ uses each one of the edge slopes in $S(t)$ exactly four times. This leads to the following equalities [3]:

$$
\begin{gathered}
n(t)=4+4 \cdot \sum_{p / q \in S(t)} 1=4 \cdot \sum_{k=1}^{t} \phi(k) \\
m(t)=1+\frac{1}{4} \cdot \operatorname{Minsum}(n(t))=1+\sum_{p / q \in S(t)}(p+q)=\sum_{k=1}^{t} k \cdot \phi(k)
\end{gathered}
$$

Here $\phi(s)$ denotes the number of integers between 1 and $s$ which are relatively prime with $s$ (the well-known Euler function from number theory;

for example

$$
\phi(1)=\phi(2)=1, \phi(3)=\phi(4)=2, \phi(5)=4 \text {.) }
$$

The summands 1 and 4 in the expressions for $m(t)$ and $n(t)$ correspond to the edges of edge slope $0 / 1$, which belong to the boundary of $M R(P(t))$.

As an illustration, "feasible" edge slopes for $t<5$, as well as the corresponding

\begin{tabular}{|c|c|c|c|c|c|c|}
\hline \multicolumn{4}{|c|}{$\begin{array}{l}\text { edge slopes }- \\
\text { members of } S(t)\end{array}$} & $t$ & $n(t)$ & $\mathrm{m}(\mathrm{t})$ \\
\hline $0 / 1$ & $0 / 1$ & $0 / 1$ & $0 / 1$ & 1 & 4 & 1 \\
\hline $1 / 1$ & $1 / 1$ & $1 / 1$ & $1 / 1$ & 2 & 8 & 3 \\
\hline $1 / 2$ & $1 / 2$ & $1 / 2$ & $1 / 2$ & & & \\
\hline $2 / 1$ & $2 / 1$ & $2 / 1$ & $2 / 1$ & 3 & 16 & 9 \\
\hline $1 / 3$ & $1 / 3$ & $1 / 3$ & $1 / 3$ & & & \\
\hline $3 / 1$ & $3 / 1$ & $3 / 1$ & $3 / 1$ & 4 & 24 & 17 \\
\hline $1 / 4$ & $1 / 4$ & $1 / 4$ & $1 / 4$ & & & \\
\hline $2 / 3$ & $2 / 3$ & $2 / 3$ & $2 / 3$ & & & \\
\hline $3 / 2$ & $3 / 2$ & $3 / 2$ & $3 / 2$ & & & \\
\hline $4 / 1$ & $4 / 1$ & $4 / 1$ & $4 / 1$ & 5 & 40 & 37 \\
\hline $1 / 5$ & $1 / 5$ & $1 / 5$ & $1 / 5$ & & & \\
\hline $5 / 1$ & $5 / 1$ & $5 / 1$ & $5 / 1$ & 6 & 48 & 49 \\
\hline
\end{tabular}
values of the functions $m(t)$ and $n(t)$, are given in Table 1 .

Table 1 
The greedy lower bound $g d l b(n)$ for arbitrary $n$ can be expressed in terms of the sequences $m(t)$ and $n(t)$ in the following way [2]:

LEMMA. If $n(t) \leqslant n<n(t+1)$, then

$$
g d l b(n)=m(t)+\lceil(n-n(t)) \cdot(t+1) / 4\rceil
$$

Proof: The perimeter of a rectangle including a digital convex $n$-gon $\boldsymbol{P}$ cannot be smaller than

$$
O=4 \cdot m(t)+(n-n(t)) \cdot(t+1)
$$

The minimal possible size of a square including $P$ is consequently equal to the smallest integer which is greater than or equal to $O / 4$.

EXAMPLE. Let $n_{1}=42$ and $n_{2}=44$. Note that $n(5)<n_{1}, n_{2}<n(6)$. The previous statement gives that $g d l b\left(n_{1}\right)=40$ and $g d l b\left(n_{2}\right)=43$. It will turn out that $F\left(n_{1}\right)=$ $1+g d l b\left(n_{1}\right)$ and $F\left(n_{2}\right)=g d l b\left(n_{2}\right)$.

It will be shown in the remaining part of this paper that the greedy lower bound for $F(n)$ is a very good one. Digital convex polygons with even number $n$ of edges either reach this bound or (in pretty rare cases) have the value of $F(n)$ equal to $g d l b(n)+1$.

\section{The MAIN RESULT}

The main result of the paper is the following explicit expression for the minimal side size $m=F(2 k)$ of a square area of the integer grid, in which a digital convex polygon with $2 k$ edges can be included, for each natural number $k$.

THEOREM 1. $F(2 k)=g d l b(2 k)$ for each integer $k \geqslant 2$, except for the following cases (a) and (b), in which $F(2 k)=1+g d l b(2 k)$

(a) $2 k=n(t)+2$,

(b) $2 k=n(t+1)-2$,

where $t$ is an odd natural number greater than 1 .

The proof of this theorem will be given in the next two sections. A construction of an optimal digital convex polygon will be described in Section 5, while the optimality of the proposed construction will be proved in Section 6 .

\section{Construction}

Throughout the remaining part of this paper, we shall assume that the integer $2 k$ satisfies the inequality $n(t) \leqslant 2 k<n(t+1)$.

When constructing optimal digital convex $2 k$-gons, we shall distinguish seven cases. 
CASE 1. $k$ is even.

An optimal digital convex $2 k$-gon can be easily constructed so that it has four equal arcs, that is, so that all its arcs have the same number of edges with the same corresponding edge slopes. The edge slopes $q / p$ within an arc are chosen so that the numbers $p$ and $q$ are relatively prime and so that the sums $p+q$ are as small as possible.

This construction can be regarded as the sum of the polygon $P(t)$ and $(2 k-n(t)) / 4$ digital squares $D S(p, q)$, where the pairs $(p, q)$ are different pairs of natural numbers $p$ and $q$, such that $p+q=t+1$.

The remaining six cases correspond to the cases when $k$ is odd.

CASE 2. $t+1=2 u+1$ for some $u \in N$

Let $Q$ denote an optimal digital convex $(2 k-2)$-gon with four equal arcs, which is constructed as in Case 1 . An optimal digital convex $2 k$-gon $P$ can be obtained from $Q$ by addition of two edges with edge slopes $u /(u+1)$, which are inserted into two opposite arcs of $Q$. It is additionally required that the edge slope $u /(u+1)$ is not used within $Q$ (equivalently, that the digital square $D S(u+1, u)$ is not used as a summand of $Q$ ).

Such an addition augments the diameter of $Q$ by exactly $u+1$.

CASE 3. $t+1=2 u$ for some $u \in N \backslash\{1\}$ and $2 k=n(t)+2$.

The construction is performed by inserting two edges with edge slope $u /(u+1)$ into two opposite arcs of $P(t)$. In this way a digital convex $2 k$-gon with diameter $m(t)+u+1$ is obtained.

CASE 4. $t+1=2 u$ for some $u \in N \backslash\{1\}$ and $2 k=n(t+1)-2$

The construction is analogous to that of Case 3. The polygon $P(t)$ should be replaced by an optimal digital convex $(n(t+1)-4)$-gon with four equal arcs and the resulting diameter is $m(t+1)-u+1$.

Two families of auxiliary digital convex 6-gons $A_{1}(w)$ and $A_{2}(w), w=2,3, \ldots$, are used for Cases 5 through 7: (Figure 2).

CASE 5. $t+1=4 w+2$ for some $w \in N$ and $2 k \in[n(t)+6, n(t+1)-6]$

An optimal digital convex $2 k$-gon is constructed as the sum of an optimal digital convex $(2 k-6)$-gon $Q$, constructed as in Case 1 and the digital convex 6-gon $A_{1}(w)$, where $Q$ has no common edge slopes with $A_{1}(w)$.

CASE 6. $t+1=4 w$ for some $w \in N$ and $2 k \in[n(t)+6, n(t+1)-10]$.

This case can be solved analogously to Case 5 , by replacing the 6-gon $A_{1}(w)$ with the 6-gon $A_{2}(w)$, the diameter of which is equal to $6 w$. 

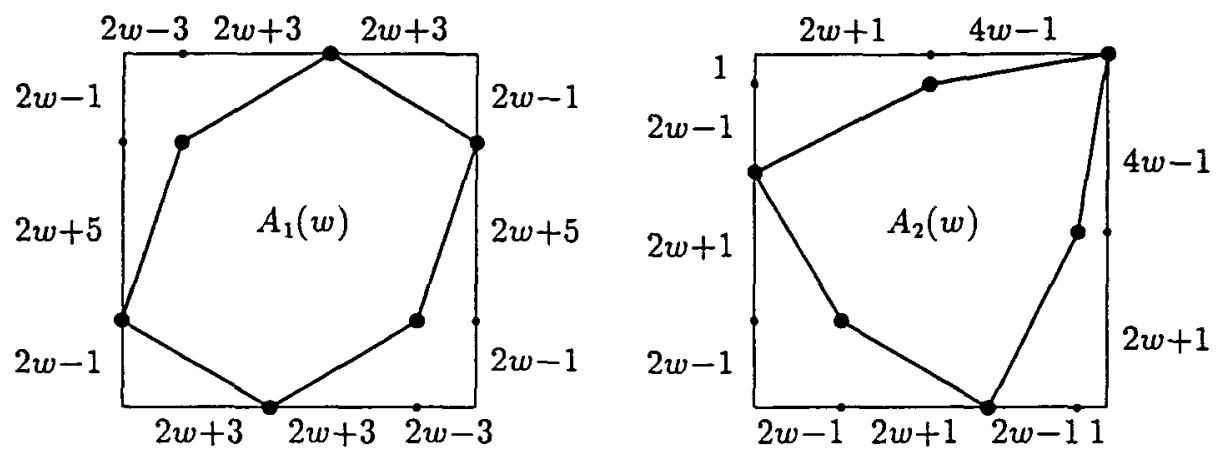

Figure 2. The families of auxiliary 6-gons

CASE 7. $t+1=4 w$ for some $w \in N$ and $2 k=n(t+1)-6$

An optimal digital convex $(n(t+1)-6)$-gon $P$ can be represented as the difference of the polygon $P(t+1)$ and the 6-gon $A_{2}(w)$. The diameter of $P$ is equal to $m(t+1)-$ $6 w$.

\section{Optimality OF THE CONSTRUCtion}

In this section we prove that the proposed construction is optimal in all the seven cases, which is equivalent to the statement that all the constructed digital convex $2 k$ gons have diameters equal to $F(2 k)$. More precisely, it will be shown that the diameter is equal to $g d l b(2 k)$ in all the cases, except for the Cases 3 and 4 , in which it is equal to $1+g d l b(2 k)$.

CASE 1. The diameter of the constructed $2 k$-gon is equal to

$$
m(t)+\frac{2 k-n(t)}{4} \cdot(t+1)=m(t)+\lceil(2 k-n(t)) \cdot(t+1) / 4\rceil=g d l b(2 k)
$$

Note that $P(t)$ is the unique digital convex $n(t)$-gon, which has diameter less or equal to $m(t)$. Namely, the only way to construct another digital convex $n(t)$-gon is to replace an edge with edge slope not greater than $t$ by an edge with edge slope greater than $t$. Such a replacement necessarily increases the perimeter of $P(t)$ by at least 1 , which implies that the diameter is also augmented by at least $[1 / 4\rceil=1$.

CASE 2. The minimal perimeter of a rectangle including some digital convex $2 k$-gon is equal to (perimeter of $Q+2 \cdot(t+1)$ ). This implies that the diameter of a digital convex $2 k$-gon cannot be smaller than the sum of diameter of $Q$ and the summand

$$
\left\lceil\frac{2 \cdot(t+1)}{4}\right\rceil=\left\lceil u+\frac{1}{2}\right\rceil=u+1 .
$$

It follows that the constructed polygon $P$ is an optimal choice. 
CASE 3. The greedy argument gives that the diameter of a digital convex $(n(t)+2)$-gon cannot be smaller than

$$
\left\lceil\frac{1}{4} \cdot(4 \cdot m(t)+2 \cdot(t+1))\right\rceil=m(t)+u
$$

We claim that this lower bound cannot be reached. Otherwise all the possible edges with bd-length not greater than $t$ must be used, together with two edges of bd-length $2 u$. If $q+p=2 u$, then $\max \{p, q\} \geqslant u+1$ (Figure 1); the edge slope $u / u$ cannot be used for $u>1$, since the edge slope $1 / 1$ has been already used. This implies that the addition of two edges with bd-length $t+1$ to the polygon $P(t)$ augments its diameter $m(t)$ at least by $u+1$. (On the contrary, observe that the addition of four such edges (with the same edge slope) can always increase the diameter by exactly $2 u$.)

CASE 4. This case is analogous to Case 3. A lower bound for the diameter, analogous to the one derived in Case 3 , is equal to

$$
\left\lceil\frac{1}{4} \cdot(4 \cdot m(t+1)-2 \cdot(t+1))\right\rceil=\lceil m(t+1)-u\rceil
$$

This lower bound cannot be reached for the same reasons as in Case 3 .

CASE 5. The diameter of a digital convex $2 k$-gon cannot be smaller than

$$
\left\lceil\frac{1}{4} \cdot(4 \cdot m(t)+(2 k-6-n(t)) \cdot(t+1))+6 \cdot(t+1)\right\rceil=\text { diameter of } Q+(6 w+3)
$$

The second summand is equal to the diameter of $A_{1}(w)$.

Note that the $(2 k-6)$-gon $Q$ cannot have more than $n(t+1)-12$ edges, since it cannot use the edges of those three digital squares $D S(p, q)$, such that the edge slopes $q / p$ are used within $A_{1}(w)$. This construction therefore cannot be applied for $2 k=n(t+1)-2$.

CASE 6. The 6-gon $A_{2}(w)$ uses four different edge-slopes, which implies that the $(2 k-6)$-gon $Q$ cannot have more than $n(t+1)-16$ edges. This was the reason for a separate treatment of Case 7.

CASE 7. The optimality of the polygon $P$ follows from the optimality of $P(t+1)$ and from the fact that $M R(P(t+1))$ and $M R\left(A_{2}(w)\right)$ are squares, which implies that $M R(P)$ is a square again.

This completes the proof of Theorem 1. 


\section{Conclusion}

The asymptotic expressions for the functions $F(n)$ and $G(m)$ were given in [10] as empirical approximation formulae.

This result was extended in [2], by proving the asymptotic estimates for $F(n)$ and $G(m)$ :

$$
F(n)=\frac{2 \pi}{12^{3 / 2}} n^{3 / 2}+O(n \log n) \quad G(m)=\frac{12}{\left(4 \pi^{2}\right)^{1 / 3}} m^{2 / 3}+O\left(m^{1 / 3} \log m\right)
$$

Theorem 1 solves the problem of determination of the exact values of $F(n)$ for even natural numbers $n$. The analogous problem for odd natural numbers $n$ still remains open, although the monotonicity of the function $F$ establishes the relatively small intervals in which these values must be located.

More precisely,

$$
F(2 k+1) \in\left[F(2 k)-1+\left\lceil\frac{t+1}{4}\right\rceil, F(2 k)+1+\left\lceil 2 \cdot \frac{t+1}{4}\right\rceil\right] .
$$

\section{APPENDIX}

The following Table 2. of the values of $g d l b(n)$ and $F(n)$ for even $n$ in $[4,120]$ is derived by using Theorem 1 .

Table 2

\begin{tabular}{|ccc|ccc|ccc|ccc|}
\hline$n$ & $g d l b$ & $F(n)$ & $n$ & $g d l b$ & $F(n)$ & $n$ & $g d l b$ & $F(n)$ & $n$ & $g d l b$ & $F(n)$ \\
\hline \hline & 1 & 1 & 32 & 27 & 27 & 62 & 74 & 74 & 92 & 132 & 132 \\
6 & 2 & 2 & 36 & 32 & 32 & 66 & 81 & 81 & 96 & 141 & 141 \\
8 & 3 & 3 & 38 & 35 & 35 & 68 & 84 & 84 & 98 & 146 & 146 \\
10 & 5 & 5 & 40 & 37 & 37 & 70 & 88 & 88 & 100 & 150 & 150 \\
12 & 6 & 6 & 42 & 40 & 41 & 72 & 91 & 91 & 102 & 155 & 155 \\
14 & 8 & 8 & 44 & 43 & 43 & 74 & 95 & 96 & 104 & 159 & 159 \\
16 & 9 & 9 & 46 & 46 & 47 & 76 & 99 & 99 & 106 & 164 & 164 \\
18 & 11 & 12 & 48 & 49 & 49 & 78 & 103 & 103 & 108 & 168 & 168 \\
20 & 13 & 13 & 50 & 53 & 53 & 80 & 107 & 107 & 110 & 173 & 173 \\
22 & 15 & 16 & 52 & 56 & 56 & 82 & 111 & 111 & 112 & 177 & 177 \\
24 & 17 & 17 & 54 & 60 & 60 & 84 & 115 & 115 & 114 & 182 & 183 \\
26 & 20 & 20 & 56 & 63 & 63 & 86 & 119 & 120 & 116 & 187 & 187 \\
28 & 22 & 22 & 58 & 67 & 67 & 88 & 123 & 123 & 118 & 192 & 192 \\
30 & 25 & 25 & 60 & 70 & 70 & 90 & 128 & 128 & 120 & 197 & 197 \\
\hline
\end{tabular}


The only differences between $g d l b(n)$ and $F(n)$ in the interval considered occur for $n=18,22,42,46,74,86$ and 114 , because of

$$
\begin{aligned}
18=n(3)+2 ; & 22=n(4)-2 ; \quad 42=n(5)+2 ; \quad 46=n(6)-2 ; \\
74=n(7)+2 ; & 86=n(8)-2 ; \quad 114=n(9)+2
\end{aligned}
$$

\section{REFERENCES}

[1] D.M. Acketa abd J.D. Žunić, 'On the number of linear partitions of the $(m, n)$-grid', Inform. Process. Lett. 38 (1991), 163-168.

[2] D. Acketa and J. Žunić, 'On the maximal number of edges of digital convex polygons included into a grid square', in Proceedings of Third Canadian Conference on Computational Geometry, 1991, pp. 215-218.

[3] D. Acketa and J. Žunić, 'A simple construction of a digital convex $2 k$-gon with minimal diameter', in Proceedings of First International Conference on the Computational Graphics and Visualization Techniques, Compugraphics 1, 1991, pp. 2-9.

[4] D. Acketa, S. Matić-Kekić and J. Žunić, 'An algorithm for a simple construction of suboptimal digital convex polygons', J. Oper. Res. Yugoslavia 2 (1992), 73-81.

[5] A. Balog and I. Barany, 'On the convex hull of the integer points in a disc', in Proceedings of Seventh Annual ACM Symposium on Computational Geometry, 1991.

[6] C.R. Guardina and E.R. Dougherty, Morphological methods in image and signal processing (Prentice Hall, Englewood Cliffs, New Jersey, 1987).

[7] D.M. Mount, and R. Silverman, 'Combinatorial and computational aspects of Minkowski decompositions', Contemp. Math. 118 (1991), 107-124.

[8] F. Preparata and M. Shamos, Computational geometry, an introduction (Springer-Verlag, Berlin, Heidelberg, New York, 1985).

[9] R.J. Simpson, 'Convex lattice polygons of minimum area', Bull. Austral. Math. Soc. 42 (1990), 353-367.

[10] K. Voss and R. Klette, 'On the maximal number of edges of a convex digital polygon included into a square', Pocitace a umela inteligencia 1 (1982), 549-558.

Institute of Mathematics

21000 Novi Sad

Trg Dositeja Obradovića 4

Serbia

Yugoslavia
Institute of Applied Basic Disciplines

Faculty of Engineering

21000 Novi Sad

Velijka Vlahovića 3, Serbia

Yugoslavia 